\title{
Exploring the limit of ENG spatio-temporal filtering for velocity-selectivity
}

\author{
Mariam Abdallah $^{1,2}$, Olivier Rossel ${ }^{1}$, Serge Bernard ${ }^{3}$, Fabien Soulier $^{2}$, and Guy Cathébras ${ }^{2}$
}

\begin{abstract}
The task of distinguishing nerve type for the activity acquired using the multi-electrode cuff presents a big interest in the possible applications of prosthesis control or functional electrical stimulation. In this paper, a method to evaluate the maximum expected performances of filters using two-dimensional spectral analysis is presented and tested on simulated extracellular potentials. These later are the result of a realistic model of an acquisition system developed from biophysiologic parameters and electrode/preamplifier model. The method is thus appropriate for characterizing filter based on velocity selective recording (VSR) method.
\end{abstract}

Index Terms-Electroneurography (ENG), nerve signal modelling, 2D-spectral analysis, velocity selective recording(VSR), multipolar cuff electrode, spatiotemporal filtering.

\section{INTRODUCTION}

Cuff electrodes which had been developed and most used in the last twenty years are established as a safe and reliable method of chronically recording the Electroneurogramm (ENG) comparing to intra-fascicular and sieve electrodes [6]. However, at present, the information extracted from each cuff is limited. Because the large number of fibers in peripheral nerves carry many neural signals, with, generally, both afferent and efferent traffic. This reduction to only one artificial signal represents a huge loss of information. Since various types of neural signal are carried by fibers of different diameters, it should be possible to extract more information from one cuff if fiber diameter-selective recording were possible [8]. This can also be seen as measuring the conduction velocity of each type of fiber, since the conduction velocity in mammalian myelinated axons is an image of the nerve structure which is described by the nodes of Ranvier, the transmembrane currents amplitude and linearly depends on the diameter [7]. So selecting particular information from the peripheral nervous system could be possible by applying the method of velocity selective recording (VSR), this technique has been used by many authors[8][3]. For this aim, multielectrode cuff (MEC) that contains typically about 10 to 15 poles presented an improvement to the method previously mentioned.

Several methods have been proposed in the literature for the estimation of conduction velocity and usually are based on a very simple idea. It consists in calculating the delay

\footnotetext{
${ }^{1}$ DEMAR team (INRIA), in LIRMM, Montpellier, France. Email: Firstname.Lastname@lirmm.fr.

${ }^{3}$ Centre National de la Recherche Scientifique,LIRMM.

${ }^{2}$ Université Montpellier,LIRMM.
}

between two or more signals registered along the direction of propagation of the action potential (AP) and then divide the distance between these two electrode contacts by the calculated delay [3]. In the case of muscle fiber, Farina et al. published in 2007 a noninvasive method for estimation of conduction velocity of conduction from EMG using a multidip approach [5]. Conduction velocity was estimated using a closed formula depending on both the temporal and spatial frequencies chosen to locate the dips. Taylor et al. also presented in many papers the improvements of the theory of VSR with spectral analysis of a single axon in an MEC with a tripolar amplifier system and the signal processing units. To improve selectivity in the velocity domain, a bandpass filter (BPF) was later inserted in the system.

However, models found in literature describe AP in the extracellular environment regardless of the presence of the structure of an electrode. Our model is developed to be able to provide the measurable signal from the nodal currents (based on the axon model) (figure 2) with respect to the electrode geometry [9]. The objective of this work is to show through simulations the maximum we can achieve in terms of distinguishing the different types of fibers, the other parts of the acquisition system being considered as "perfect".

This paper has three major sections. We first present the biophysiologic and electrode models needed for simulation. Spectral analysis of the simulation results in the spatiotemporal domains are also studied. They show not only the velocity of each type of myelinated fiber but also the relationship between the velocity and the distance between two successive nodes of Ranvier (internode). Second, results obtained for different filter characteristics are treated and presented. And third, the limits and the implications of these results are examined in the discussion.

\section{Methods}

An ENG acquisition system as showed in figure 1 is modeled. It is based on a MEC to achieve discrimination of conduction velocities and then select one type fiber activity. Biophysiologic model is also used to provide the extracellular biopotentials. The model of the electrode is built around realistic parameters such as size and number of poles and inter-poles distance ( pitch) that are usually constrained due to anatomical particularities and expected amplitude of the recorded signal [1]. This system is generally designed to be able to select the information of one type of fiber. We include 


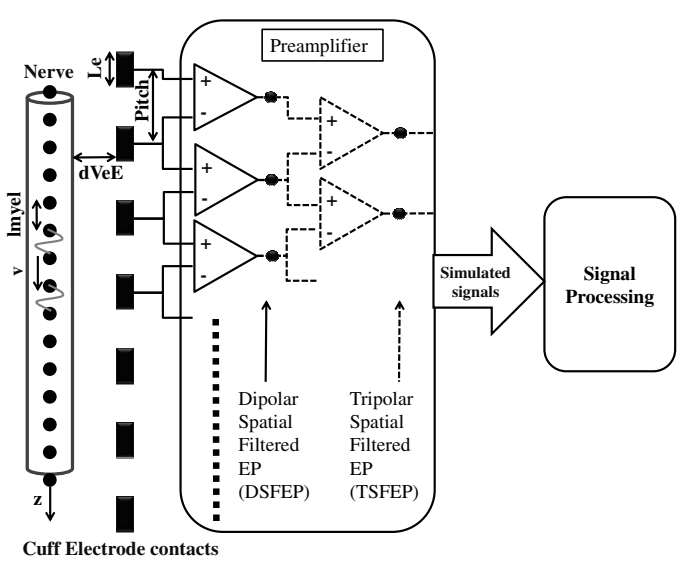

Figure 1. Prototype of an ENG acquisition system.

a preamplifier system together with the electrode cuff model to be able to test spatiotemporal filters against realistic signals and not those that would be measured on the surface of the nerve.

\section{A. Modelization}

Figure 2 presents the different steps for modelization starting from the biophysiological model in the first block, electrode model and preamplifier model in the second block to get finally the simulated signals that should be processed.

1) Biophysiological Model: The biophysical model implements a model proposed by Durand et al. [10] and takes into consideration the characteristics of the fibers constituting the nerve. It considers only myelinated axons, where $l_{\text {myel }}$ is the internodal length (i.e. the distance between two successive Ranvier nodes), the axon diameter and the fiber diameter. We chose to make $l_{\text {myel }}$ vary from 0.5 to $2 \mathrm{~mm}$, with fiber diameter varying from 5 to $20 \mu \mathrm{m}$. According to the study by Durand et al. [10], there is a 0.6 ratio between the diameter of the axon and the diameter of the fiber. Plus, there is a 100 ratio between the internodal length and the fiber diameter [10]. The following simulation results focuses on four types of fiber. The potential is obtained from a the nodal currents by using a homogenous isotropic extracellular model. Thus, a spatial transfer function allows to compute extracellular potential over time and space $\left(V_{e}\right)$.

2) Electrode/Preamplifier Model: This part of model (second block on figure 2) takes the number and the distance between cuff electrode contacts (pitch) as a parameter. It also take into account the size of the electrode contact $L e$ which. This effect is modeled by applying spatial averaging over $V e$. This point is generally not modeled in other publications while it could be controlled in our model. Finally, the preamplifier frontend usually uses dipolar or tripolar configuration. We chose to implement both and during simulation, we have the choice to select one or another. At last, the model provides simulated signals as an output to be processed.

\section{B. Spectral Analysis Of Simulated Signals}

Since we decided to study the velocity selectivity, spectral analysis of the simulated signals from the preamplifier (dipolar or tripolar) stage was performed to yield information about the conduction velocity of the associated nerve fiber.

Spectral analysis can be carried out on the simulated signals either in temporal domain, in spatial domain or both simultaneously. In the direction of the propagation of the signal, the spatial frequency $f_{z}$ domain is related to the temporal frequency $f_{t}$ domain by the velocity of propagation which also refers to the interpole distance $d$ and the delay $T$ between the appearance of the signals at successive measurements points :

$$
v=\frac{f_{t}}{f_{z}}=\frac{d}{T}
$$

For one-dimension spectral analysis, we used the 1D Discret Fourier Transform. Two-dimensional spectral analysis of simulated signals is also substantial to show how spatiotemporal filtering techniques can improve the selectivity. In that case, the simulated signals modeled as mentioned before were spectrally transformed using a 2D Discrete Fourier Transform (2D-DFT):

$$
S\left(f_{z}, f_{t}\right)=\frac{1}{M N} \sum_{z=1}^{M} \sum_{t=1}^{N} s(z, t) e^{-i 2 \pi\left(f_{s} \frac{z}{M}+f_{t} \frac{t}{N}\right)}
$$

where $s(z, t)$ is the spatiotemporal simulated signal and $S\left(f_{z}, f_{t}\right)$ is the signal in the $f_{z}$ and $f_{t}$ spatial and temporal frequencies. $S p$ is the spectral power in $\mathrm{dB}$ and can be written as follows: $S p=20 \log _{10}(|S|)$.

The output of a Fourier transform comprises positive and negative frequencies. We chose to represent centered spectrum $\left(\left(f_{z}=0, f_{t}=0\right)\right.$ in the center of plot $)$ in the next figures.

\section{Optimal Selectivity}

Our objective will be thus to select one or another fiber by applying an optimal filter on the main lobe of each spectrum. The first step is to select the energy in the main lobe of the spectrum corresponding to a certain velocity of propagation. This is equivalent to apply spatio-temporal filtering in the velocity domain $(z, t)$.

By applying a filter mask $H\left(f_{z}, f_{t}\right)$ on the $2 \mathrm{D}$ spectrum, we estimate the electrical signals resulting from the simulated signals after filtering. This process is done by using by the inverse Fourier transform (IFFT):

$$
v(z, t)=\sum_{f_{z}=1}^{M} \sum_{f_{t}=1}^{N} V\left(f_{z}, f_{t}\right) e^{i 2 \pi\left(f_{s} \frac{z}{M}+f_{t} \frac{t}{N}\right)}
$$

where $v(z, t)$ is the ideally filtered output signal and $V\left(f_{z}, f_{t}\right)=H\left(f_{z}, f_{t}\right) \cdot S\left(f_{z}, f_{t}\right)$ is the selected data from the $2 \mathrm{D}$ spectrum.

\section{Simulated Results}

\section{A. Simulation Setup}

Myelinated peripheral nerve fibers are classified on the basis of fiber diameter and velocity of conduction of nerve 


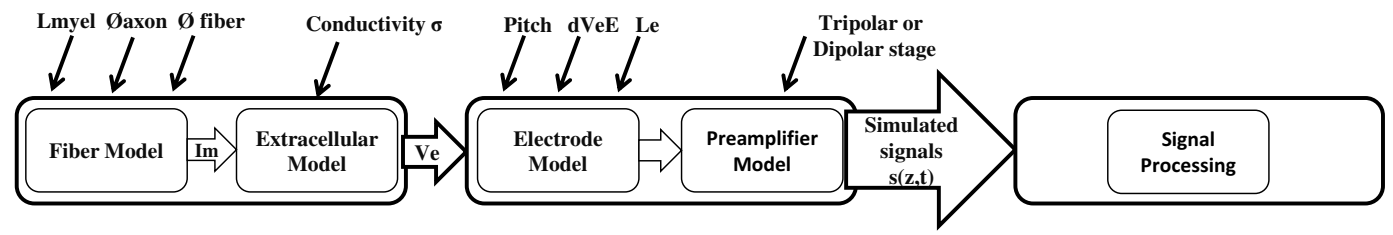

Figure 2. Block diagram of the proposed acquisition chain. Membrane currents and extracellular voltage are computed and the signals are simulated in order to apply spectral analysis.
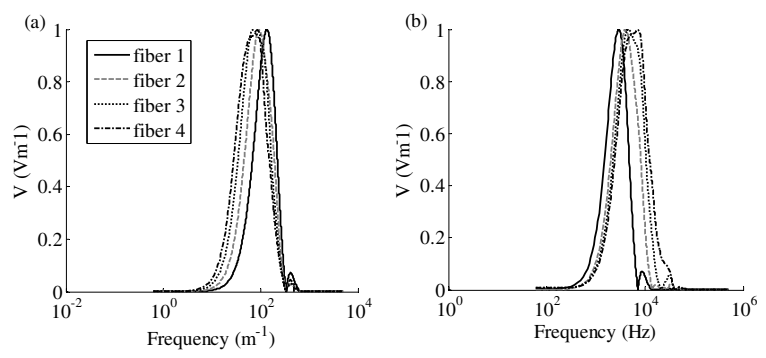

Figure 3. Spectral and temporal 1D spectrum for four simulated signals presenting four types of fibers.

impulses. The velocity of conduction begin with $3 \mathrm{~m} / \mathrm{s}$ and can reach $120 \mathrm{~m} / \mathrm{s}$ [4]. The simulation setup is based on the different type of fibers. We chose for the simulation only four types of fibers, described by their diameter range from 5 to $20 \mu \mathrm{m}$ and velocity of propagation range from 20 to $84 \mathrm{~m} / \mathrm{s}$ as shown in table I. These four velocities are representative of types I, II, III of mammalian afferent fibers.

Concerning the electrode, the contact size $L e$ is considered $1 \mathrm{~mm}$, the pitch $3 \mathrm{~mm}$, that is compatible with state-of-the-art MEC. The distance between the contact and the axon is set to $1 \mathrm{~mm}$ and the preamplifier stage is in tripolar configuration.

\section{B. Temporal or Spatial Filtering}

Figure 3 (a) represents the spatial spectrum of simulated signals from the preamplifier, and figure 3 (b) presents the temporal spectrums. Both of them are associated to the four types of fibers. As one can observe, the maximum of energy in both spectrum belong almost to the same range of frequencies, regardless of the fiber type. This fact ilustrates that a simple processing of the simulated signals in the time domain for a single acquisition point is not sufficient to discriminate the characteristics of the fiber. Similarly, spatial analysis at a given time is not sufficient either. In other terms, this would mean that the one dimension filters are not able to differentiate the characteristics of the extracellular signals coming from different fiber types.

\section{Spatiotemporal Filtering}

As shown in the figure 4, two-dimensional Fourier Transform applied on the simulated signals reveals significant differences in the spatio-temporal distribution of the energy depending on the fiber characteristics. Most of the energy spreads on the main lobe of the spectrum that is oriented
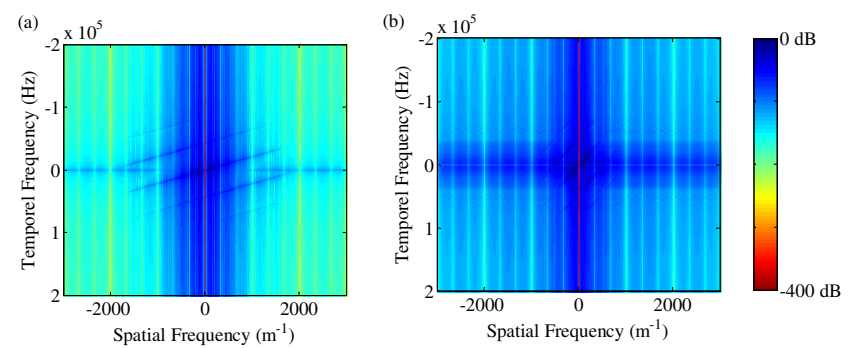

Figure 4. 2D Spectrum of the 2D FFT for extracellular signal representing the fiber type one (a) and four (b).

according to the velocity of propagation, related to equation 1.

Thus, optimal velocity filters must be designed to select only this main lobe, letting other values to zero. Another interesting property is the periodic minimums observed vertically, related to the spatial sampling frequency of the electrode : $F_{n}=\frac{n}{d}, n \in \mathbb{Z}$

Minimums related to the pole width are also incorporated in the spectrum at the $K_{n}: K_{n}=\frac{n}{L_{e}}, n \in \mathbb{Z}$

Moreover, a pseudo-periodicity due to the discrete nature of nodal currents is linked to internode distance $l_{\text {myel }}$ such as: $I_{n}=\frac{n}{l_{\text {myel }}}, n \in \mathbb{Z}$

Finally, the low-frequency spectral energy indicates the global variations due to the signal propagation along the axon. These parameters can be used to carry out fiber information and help to design selective filters.

\section{Results for optimal spatio-temporal filters}

Figure 5 reveals the simulated signals before processing. We can clearly see the difference between the amplitudes generated by each type of fiber. Fiber 4 , characterized by a velocity of $84 \mathrm{~m} / \mathrm{s}$ presents a higher signal amplitude (b).

We designed an ideal filtering so that the 2D transfer function selects only the direction related to the chosen velocity. The figure 6 gives the output result of such filtered signals. Filter used in (a) and (b) is selective for the fiber type one and attenuates the others. Similarly, the filter used in (c) and (d) decreases the fiber-signal amplitude except for the signal corresponding to the fiber-type four.

To get objective selectivity measure, we introduce the energy criteria calculated from output signals. Table I presents the energy criteria of the optimal 2D filtering for each fiber according to each filter. Every filter is selective for a 

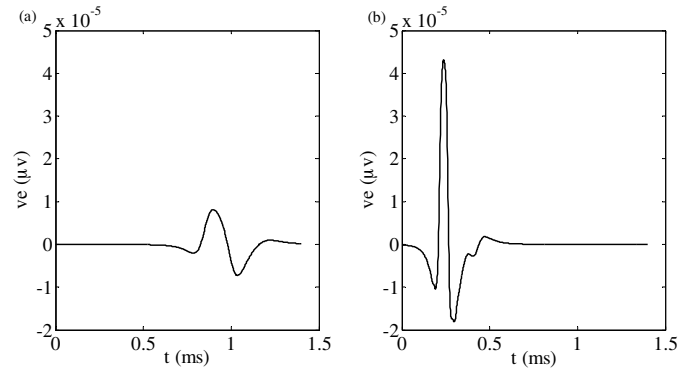

Figure 5. Extracellular signals before processing for the type of fiber one (a) and four (b)

\begin{tabular}{c|c|c|c|c}
\hline & Fiber 1 & Fiber 2 & Fiber 3 & Fiber 4 \\
\hline Velocity $(\mathrm{m} / \mathrm{s})$ & 20.0506 & 40.2499 & 63.6312 & 83.3821 \\
\hline Diameter $(\mu \mathrm{m})$ & 5 & 10 & 15 & 20 \\
\hline Energy criteria & Fiber 1 & Fiber 2 & Fiber 3 & Fiber 4 \\
\hline Filter 1 & 3.102 & 0.2806 & 0.2234 & 0.2809 \\
\hline Filter 2 & 0.0246 & 20.062 & 0.8511 & 0.5685 \\
\hline Filter 3 & 0.0040 & 0.2678 & 55.88 & 3.8713 \\
\hline Filter 4 & 0.0019 & 0.0652 & 0.8555 & 79.258 \\
\hline
\end{tabular}

Table I

CALCULATED ENERGY FOR EACH FILTER ASSOCIATED TO A SPATIAL VELOCITY.

certain velocity of propagation and, as expected, presents a maximum energy for the signal related to the corresponding fiber. For example, filter 4 is selective for fiber four. It has a maximum energy (79.258) related to velocity of $84 \mathrm{~m} / \mathrm{s}$ and decreases all other signal amplitudes.

\section{Discussion}

It has to be noticed that fibers do not provide the same signal amplitude since the amplitude of an action potential recorded extracellularly is proportional to the square of the conduction velocity of the fiber [2]. In addition to this, in physiological context, the neural signal is rather a compound ENG than a single action potential. So even if we have a good selectivity for filters tuned to the slow velocities it could be hidden with the amplitude of the fibers with high velocities.

The solution proposed to this problem would be possible by parallel processing of a compound ENG from different fibers by a bank of filters, each matched to a specific velocities. By comparing the output of the filters, one can evaluate the ratio of each fiber type firing at a time.

Another limitation is that the results presented in the table I show the ideal filter matched to a population of fibers. The realistic velocity filter would be less selective because we are limited in term of number of poles for the cuff electrode and the filter coefficients.

\section{Conclusions}

This paper explores the ideal selectivity that can be reached by velocity filters. One dimensional spectral analysis were applied on simulated signals and results proved that onedimensionnal processing is not sufficient. Two-dimensional
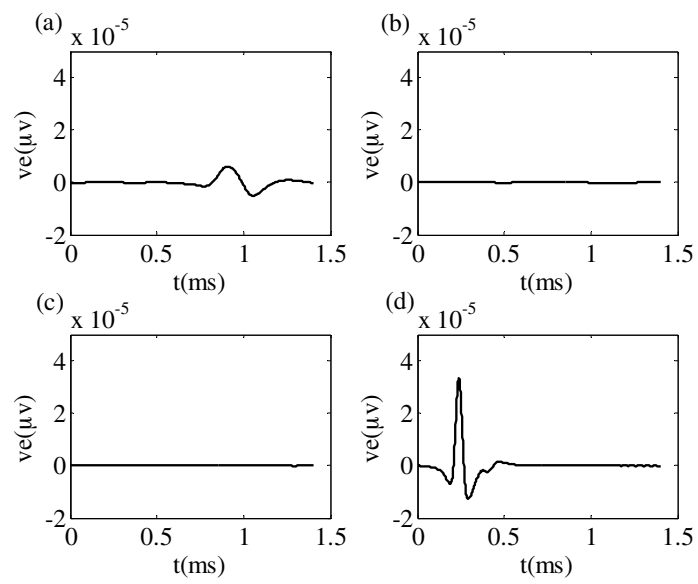

Figure 6. Filtered Extracellular signals with an ideal filter related to a given velocity (a) output of filter 1 applied on fiber 1 (b) output of filter 1 applied on fiber 4 (c) output of filter 4 applied on fiber 1 and (d) output of filter 4 applied on fiber 4 .

spectral analysis were then studied on simulated signals. It revealed that properties of axons defines spectral characteristics of different recorded signals. Based on these analyzes, it was possible to define an ideal two-dimensional filter related to a group of velocities. We then introduced the energy criteria that make it possible to evaluate the maximum selectivity one can expect for spatio-temporal filtering of nerve fibers signals.

\section{REFERENCES}

[1] L.N.S. Andreasen and J.J. Struijk. Signal Strength Versus Cuff Length in Nerve Cuff Electrode Recordings. IEEE Transac on Biomedical Engineering, 49(9):1045-1050, 2002.

[2] I.A. Boyd and K.U. Kalu. Physiology, University of Glasgow, Glasgow 012. pages 277-297, 1979.

[3] C.T. Clarke, X. Xu, R. Rieger, J. Taylor, and N. Donaldson. An implanted system for multi-site nerve cuff-based ENG recording using velocity selectivity. Analog Integrated Circuits and Signal Processing, 58(2):91-104, 2008.

[4] N. Donaldson, R. Rieger, M. Schuettler, and J. Taylor. Noise and selectivity of velocity-selective multi-electrode nerve cuffs. Medical \& biological engineering \& computing, 46(10):1005-18, October 2008.

[5] D. Farina and F. Negro. Estimation of Muscle Fiber Conduction Velocity with a Spectral Multidip Approach. IEEE Transactions on Biomedical Engineering, 54(9):1583-1589, 2007.

[6] T. Kawada, C. Zheng, S. Tanabe, T. Uemura, K. Sunagawa, and M. Sugimachi. A sieve electrode as a potential autonomic neural interface for bionic medicine. Annual International Conference of the IEE Engineering in Medecine and Biology Society., 6:4318-21, January 2004.

[7] J. Malmivuo and R. Plonsey. Bioelectromagnetism Principles and Applications of Bioelectric and Biomagnetic Fields. New York Oxford Oxford University Press, 1995.

[8] R Rieger, J. Taylor, E. Comi, N. Donaldson, M. Russold, C.M.O. Mahony, J.A. McLaughlin, E. McAdams, A. Demosthenous, and J.C. Jarvis. Experimental determination of compound action potential direction and propagation velocity from multi-electrode nerve cuffs. Medical engineering \& physics, 26(6):531-4, July 2004.

[9] O. Rossel, F. Soulier, S. Bernard, and G. Cathébras. New Electrode Layout for Internal Selectivity of Nerves. In EMBC09: 31st Annual International Conference of the IEEE Engineering in Medicine and Biology Society, 2009.

[10] E.N. Warman, W.M. Grill, and D. Durand. Modeling the effects of electric fields on nerve fibers: determination of excitation thresholds. IEEE transactions on bio-medical engineering, 39(12):1244-54, December 1992. 